\title{
SANDWICH RESULTS FOR HIGHER ORDER FRACTIONAL DERIVATIVE OPERATORS
}

H. M. Zayed, T. Bulboacă. Sandwich results for higher order fractional derivative operators, Mat. Stud. 49 (2018), 52-66.

In this paper we obtain some differential subordinations and superordinations related to a generalized fractional derivative operator for higher order derivatives of multivalent functions. Moreover, we derive some sandwich results under certain assumptions on the parameters involved, and these new results generalize some previously well-known theorems.

\section{In memory of Professor Yuri Borisovich Zelinskii}

1. Introduction. Let $\mathcal{H}(\mathbb{U})$ denote the space of analytic functions in $\mathbb{U}:=\{z \in \mathbb{C}:|z|<1\}$ and let $\mathcal{H}[a, p]$ denote the subclass of functions $f \in \mathcal{H}(\mathbb{U})$ of the form

$$
f(z)=a+a_{p} z^{p}+a_{p+1} z^{p+1}+\ldots, z \in \mathbb{U} \quad(a \in \mathbb{C}, p \in \mathbb{N}=\{1,2, \ldots\}) .
$$

Also, let $\mathcal{A}(p)$ denote the subclass of multivalent functions $f \in \mathcal{H}(\mathbb{U})$ with the power series expansion

$$
f(z)=z^{p}+\sum_{n=1}^{\infty} a_{p+n} z^{p+n}, \quad z \in \mathbb{U} \quad(p \in \mathbb{N}) .
$$

For two functions $f, g \in \mathcal{H}(\mathbb{U})$, we say that the function $f$ is subordinate to $g$, written $f \prec g$, if there exists a Schwarz function $w$, analytic in $\mathbb{U}$, with $w(0)=0$ and $|w(z)|<1$, such that $f(z)=g(w(z))$ for all $z \in \mathbb{U}$.

Furthermore, if the function $g$ is univalent in $\mathbb{U}$, then we have the following equivalence (see $[9,11])$ :

$$
f \prec g \Leftrightarrow(f(0)=g(0) \quad \text { and } \quad f(\mathbb{U}) \subset g(\mathbb{U})) .
$$

Let $\phi(r, s, t ; z): \mathbb{C}^{3} \times \mathbb{U} \rightarrow \mathbb{C}$ and $h$ be univalent in $\mathbb{U}$. If $p$ is analytic in $\mathbb{U}$ and satisfies the second order differential subordination

$$
\phi\left(p(z), z p^{\prime}(z), z^{2} p^{\prime \prime}(z) ; z\right) \prec h(z), z \in \mathbb{U},
$$

then $p$ is a solution of the differential subordination (2). The univalent function $q$ is said to be a dominant of (2) if $p \prec q$ for all $p$ that satisfying (2). A dominant $\widetilde{q}$ is called the best dominant if $\widetilde{q} \prec q$ for all dominants $q$.

2010 Mathematics Subject Classification: 30C45, 30C55, 30C80.

Keywords: analytic functions; univalent functions; differential subordination and superordination; hypergeometric function; generalized fractional derivative operator.

doi:10.15330/ms.49.1.52-66

(C) H. M. Zayed, T. Bulboacă, 2018 
Let $\varphi(r, s, t ; z): \mathbb{C}^{3} \times \mathbb{U} \rightarrow \mathbb{C}$ and $h$ be analytic in $\mathbb{U}$. If $p$ and $\varphi\left(p(z), z p^{\prime}(z), z^{2} p^{\prime \prime}(z) ; z\right)$ are univalent functions and if $p$ satisfies the second order differential superordination

$$
h(z) \prec \varphi\left(p(z), z p^{\prime}(z), z^{2} p^{\prime \prime}(z) ; z\right), z \in \mathbb{U},
$$

then $p$ is a solution of the differential superordination (3). An analytic function $q$ is said to be a subordinant of (3) if $q \prec p$ for all $p$ satisfies (3). A univalent subordinant $\widetilde{q}$ that satisfies $q \prec \widetilde{q}$ for all subordinants $q$ of (3) is said to be the best subordinant. Recently, Miller and Mocanu ([12]) obtained conditions on $h, q$ and $\varphi\left(p(z), z p^{\prime}(z), z^{2} p^{\prime \prime}(z) ; z\right)$ for which the following implication holds:

$$
h(z) \prec \varphi\left(p(z), z p^{\prime}(z), z^{2} p^{\prime \prime}(z) ; z\right) \Longrightarrow q(z) \prec p(z), z \in \mathbb{U} .
$$

Following Miller and Mocanu ([12]), Bulboacă ([7]) investigated certain classes of first order differential superordinations as well as superordination preserving integral operators ([8]). Ali et al. [1] used the results obtained by Bulboacă ([8]) and gave the sufficient conditions for certain normalized analytic functions $f$ to satisfy

$$
q_{1}(z) \prec \frac{f(z)}{z f^{\prime}(z)} \prec q_{2}(z), z \in \mathbb{U},
$$

where $q_{1}$ and $q_{2}$ are given univalent functions in $\mathbb{U}$ with $q_{1}(0)=q_{2}(0)=1$.

Shanmugam et al. [16] obtained sufficient conditions for normalized analytic functions to satisfy

$$
q_{1}(z) \prec \frac{f(z)}{z f^{\prime}(z)} \prec q_{2}(z), \quad q_{1}(z) \prec \frac{z f^{\prime}(z)}{f^{2}(z)} \prec q_{2}(z), z \in \mathbb{U},
$$

where $q_{1}$ and $q_{2}$ are univalent functions in $\mathbb{U}$ with $q_{1}(0)=q_{2}(0)=1$.

Note that for a function $f \in \mathcal{A}(p)$ of the form (1) the $m$-th order derivative of $f$ could be written as

$$
\begin{gathered}
f^{(m)}(z)=\delta(p, m) z^{p-m}+\sum_{n=1}^{\infty} \delta(p+n, m) a_{p+n} z^{p+n-m}, z \in \mathbb{U} \\
\left(p>m, m \in \mathbb{N}_{0}:=\mathbb{N} \cup\{0\}\right),
\end{gathered}
$$

where

$$
\delta(p, m)=\frac{p !}{(p-m) !}:= \begin{cases}p(p-1) \ldots(p-m+1), & \text { if } m \neq 0 \\ 1, & \text { if } m=0 .\end{cases}
$$

Let ${ }_{2} F_{1}(a, b ; c ; z)$ be the well-known (Gaussian) hypergeometric function defined by

$$
{ }_{2} F_{1}(a, b ; c ; z):=\sum_{n=0}^{\infty} \frac{(a)_{n}(b)_{n}}{(c)_{n}(1)_{n}} z^{n}, z \in \mathbb{U}
$$

where $c \neq 0,-1,-2, \ldots$ and

$$
(\lambda)_{n}:= \begin{cases}1, & \text { if } n=0, \\ \lambda(\lambda+1)(\lambda+2) \ldots(\lambda+n-1), & \text { if } n \in \mathbb{N} .\end{cases}
$$

We will recall some definitions and lemmas which will be used in our paper. 
Definition 1 ([14]). The fractional derivative of order $\lambda$ for a function $f$ is defined by

$$
D_{z}^{\lambda} f(z):=\frac{1}{\Gamma(1-\lambda)} \frac{d}{d z} \int_{0}^{z} \frac{f(\zeta)}{(z-\zeta)^{\lambda}} d \zeta \quad(0 \leq \lambda<1), z \in \mathbb{U},
$$

where $f$ is analytic in a simply-connected region of the complex $z$-plane containing the origin and the multiplicity of $(z-\zeta)^{-\lambda}$ is removed by requiring $\log (z-\zeta)$ to be real when $z-\zeta>0$.

In this paper we define the generalized fractional derivative operator for higher order derivative of multivalent functions as follows:

Definition 2. Assume that $0 \leq \lambda<1, \mu, \eta \in \mathbb{R}$ and $m \in \mathbb{N}_{0}$. Then, the generalized fractional derivative operator of order $m$ for a function $f \in \mathcal{A}(p)$ is given by

$$
\begin{gathered}
\mathrm{J}_{0, z}^{\lambda, \mu, \eta, p}(m) f(z):= \\
:=\frac{d}{d z}\left[\frac{z^{\lambda-\mu}}{\Gamma(1-\lambda)} \int_{0}^{z}(z-\zeta)^{-\lambda} f^{(m)}(\zeta)_{2} F_{1}\left(\mu+\lambda, 1-\eta ; 1-\lambda ; 1-\frac{\zeta}{z}\right) d \zeta\right], z \in \mathbb{U},
\end{gathered}
$$

where $f$ is an analytic function in a simply-connected region of the complex $z$-plane containing the origin with the order $f(z)=O\left(|z|^{\varepsilon}\right), z \rightarrow 0$ when $\varepsilon>\max \{0, \mu-\eta\}-1$, and the multiplicity of $(z-\zeta)^{-\lambda}$ is removed by requiring $\log (z-\zeta)$ to be real when $z-\zeta>0$.

Remark 1. (i) Note that $J_{0, z}^{\lambda, \mu, \eta, p}(0)=J_{0, z}^{\lambda, \mu, \eta, p}$ ([2]- [6], [13]).

(ii) Under the hypotheses of Definition 2 we have

$$
\mathrm{J}_{0, z}^{\lambda+k, \mu+k, \eta+k, p}(m) f(z)=z^{k} \frac{d^{k}}{d z^{k}} \mathrm{~J}_{0, z}^{\lambda, \mu, \eta, p}(m) f(z) .
$$

Note that $J_{0, z}^{\lambda, \lambda, \eta, p}=D_{z}^{\lambda}$, and denote

$$
\mathrm{D}_{z}^{\lambda}(m):=\mathrm{J}_{0, z}^{\lambda, \lambda, \eta, p}(m)
$$
by

Goyal and Prajapat ([10], see also $[2-4,6])$ defined the operator $\mathcal{M}_{0, z}^{\lambda, \mu, \eta, p}: \mathcal{A}(p) \rightarrow \mathcal{A}(p)$

$$
\begin{gathered}
\mathcal{M}_{0, z}^{\lambda, \mu, \eta, p} f(z):=\frac{\Gamma(p+1-\mu) \Gamma(p+1-\lambda+\eta)}{\Gamma(p+1) \Gamma(p+1-\mu+\eta)} z^{\mu} J_{0, z}^{\lambda, \mu, \eta, p} f(z) \\
=z^{p}+\sum_{n=1}^{\infty} \frac{(p+1)_{n}(p+1-\mu+\eta)_{n}}{(p+1-\mu)_{n}(p+1-\lambda+\eta)_{n}} a_{p+n} z^{p+n}, z \in \mathbb{U}, \\
(0 \leq \lambda<1, \mu<p+1, \eta>\max \{\lambda, \mu\}-p-1),
\end{gathered}
$$

where $f \in \mathcal{A}(p)$ has the form (1).

Let now define the operator $\mathrm{M}_{0, z}^{\lambda, \mu, \eta, p}(m): \mathcal{A}(p) \rightarrow \mathcal{A}(p-m)$ as follows

$$
\begin{gathered}
\mathrm{M}_{0, z}^{\lambda, \mu, \eta, p}(m) f(z):=\frac{\Gamma(p-m+1-\mu) \Gamma(p-m+1-\lambda+\eta)}{\Gamma(p-m+1) \Gamma(p-m+1-\mu+\eta)} z^{\mu} \mathrm{J}_{0, z}^{\lambda, \mu, \eta, p}(m) f(z)= \\
=\delta(p, m) z^{p-m}+\sum_{n=1}^{\infty} \delta(p+n, m) \Psi_{n}(p, m, \lambda, \mu, \eta) a_{p+n} z^{p+n-m}, z \in \mathbb{U},
\end{gathered}
$$




$$
(0 \leq \lambda<1, \mu<p-m+1, \eta>\max (\lambda, \mu)-p+m-1)
$$

where

$$
\Psi_{n}(p, m, \lambda, \mu, \eta):=\frac{(p-m+1)_{n}(p-m+1-\mu+\eta)_{n}}{(p-m+1-\mu)_{n}(p-m+1-\lambda+\eta)_{n}},
$$

and $f \in \mathcal{A}(p)$ has the power series expansion (1).

Remark 2. Note that $\mathrm{M}_{0, z}^{\lambda, \mu, \eta, p}(0)=\mathcal{M}_{0, z}^{\lambda, \mu, \eta, p}$ (Goyal and Prajapat [10]). It is easy to verify that for $z \in \mathbb{U}$,

$$
z\left(\mathrm{M}_{0, z}^{\lambda, \mu, \eta, p}(m) f(z)\right)^{\prime}=(p-m-\mu) \mathrm{M}_{0, z}^{\lambda+1, \mu+1, \eta+1, p}(m) f(z)+\mu \mathrm{M}_{0, z}^{\lambda, \mu, \eta, p}(m) f(z) .
$$

Definition 3 ([11]). Denote by $\mathcal{Q}$ the set of all functions $f$ that are analytic and univalent on $\overline{\mathbb{U}} \backslash E(f)$, where

$$
E(f):=\left\{\varsigma \in \partial \mathbb{U}: \lim _{z \rightarrow \varsigma} f(z)=\infty\right\},
$$

and are such that $f^{\prime}(\varsigma) \neq 0$ for $\varsigma \in \partial \mathbb{U} \backslash E(f)$.

Lemma 1 ([11]). Let $q$ be a univalent function in $\mathbb{U}$ and $\theta$ and $\varphi$ be analytic in a domain $\mathcal{D}$ containing $q(\mathbb{U})$, with $\varphi(w) \neq 0$ when $w \in q(\mathbb{U})$. Set

$$
\psi(z)=z q^{\prime}(z) \varphi(q(z)) \quad \text { and } \quad h(z)=\theta(q(z))+\psi(z), z \in \mathbb{U} .
$$

Suppose that

(i) $\psi$ is starlike univalent in $\mathbb{U}$,

(ii) $\operatorname{Re} \frac{z h^{\prime}(z)}{\psi(z)}>0, z \in \mathbb{U}$.

If $p$ is analytic with $p(0)=q(0), p(\mathbb{U}) \subset \mathcal{D}$ and

$$
\theta(p(z))+z p^{\prime}(z) \varphi(p(z)) \prec \theta(q(z))+z q^{\prime}(z) \varphi(q(z)), z \in \mathbb{U},
$$

then $p \prec q$ and $q$ is the best dominant.

Lemma 2 ([7]). Let $q$ be a univalent function in $\mathbb{U}$ and $\theta$ and $\varphi$ be analytic in a domain $\mathcal{D}$ containing $q(\mathbb{U})$. Suppose that

(i) $\operatorname{Re} \frac{\theta^{\prime}(q(z))}{\varphi(q(z))}>0, z \in \mathbb{U}$,

(ii) $\psi(z)=z q^{\prime}(z) \varphi(q(z))$ is starlike univalent in $\mathbb{U}$.

If $p \in \mathcal{H}[q(0), 1] \cap \mathcal{Q}$ with $p(\mathbb{U}) \subset \mathcal{D}, \theta(p(z))+z p^{\prime}(z) \varphi(p(z))$ is univalent in $\mathbb{U}$ and

$$
\theta(q(z))+z q^{\prime}(z) \varphi(q(z)) \prec \theta(p(z))+z p^{\prime}(z) \varphi(p(z)), z \in \mathbb{U},
$$

then $q \prec p$ and $q$ is the best subordinant.

Lemma 3 ([15]). The function $q(z)=(1-z)^{-2 a b}, z \in \mathbb{U}$, is univalent in $\mathbb{U}$ if and only if $|2 a b-1| \leq 1$ or $|2 a b+1| \leq 1$.

2. Subordination results. Unless otherwise mentioned, we assume throughout this paper that $0 \leq \lambda<1, \mu<p-m+1, \eta>\max \{\lambda ; \mu\}-p+m-1, m \in \mathbb{N}_{0}, m<p, p \in \mathbb{N}$, and denote $\mathbb{C}^{*}:=\mathbb{C} \backslash\{0\}$. 
Theorem 1. Let $q$ be univalent in $\mathbb{U}$ with $q(0)=1, q(z) \neq 0$ for all $z \in \mathbb{U}$ and $\frac{z q^{\prime}(z)}{q(z)}$ be starlike univalent in $\mathbb{U}$, such that

$$
\operatorname{Re}\left[1+\frac{\beta}{\zeta} q(z)+\frac{2 \gamma}{\zeta} q^{2}(z)+\frac{z q^{\prime \prime}(z)}{q^{\prime}(z)}-\frac{z q^{\prime}(z)}{q(z)}\right]>0, z \in \mathbb{U},\left(\beta, \gamma \in \mathbb{C}, \zeta \in \mathbb{C}^{*}\right) .
$$

Let $f \in \mathcal{A}(p)$ and for $z \in \mathbb{U}$,

$$
\begin{aligned}
& \Omega_{\lambda, \mu, \eta, p, m}(\beta, \gamma, \zeta) f(z):=\beta \frac{\mathrm{M}_{0, z}^{\lambda, \mu, \eta, p}(m) f(z)}{\mathrm{M}_{0, z}^{\lambda+1, \mu+1, \eta+1, p}(m) f(z)}+\gamma\left(\frac{\mathrm{M}_{0, z}^{\lambda, \mu, \eta, p}(m) f(z)}{\mathrm{M}_{0, z}^{\lambda+1, \mu+1, \eta+1, p}(m) f(z)}\right)^{2}+ \\
+ & \zeta\left[(p-m-\mu) \frac{\mathrm{M}_{0, z}^{\lambda+1, \mu+1, \eta+1, p}(m) f(z)}{\mathrm{M}_{0, z}^{\lambda, \mu, \eta, p}(m) f(z)}-(p-m-\mu-1) \frac{\mathrm{M}_{0, z}^{\lambda+2, \mu+2, \eta+2, p}(m) f(z)}{\mathrm{M}_{0, z}^{\lambda+1, \mu+1, \eta+1, p}(m) f(z)}-1\right] .
\end{aligned}
$$

If

$$
\Omega_{\lambda, \mu, \eta, p, m}(\beta, \gamma, \zeta) f(z) \prec \beta q(z)+\gamma q^{2}(z)+\zeta \frac{z q^{\prime}(z)}{q(z)}, z \in \mathbb{U},
$$

then

$$
\frac{\mathrm{M}_{0, z}^{\lambda, \mu, \eta, p}(m) f(z)}{\mathrm{M}_{0, z}^{\lambda+1, \mu+1, \eta+1, p}(m) f(z)} \prec q(z), z \in \mathbb{U},
$$

and $q$ is the best dominant of (8).

Proof. Let

$$
p(z)=\frac{\mathrm{M}_{0, z}^{\lambda, \mu, \eta, p}(m) f(z)}{\mathrm{M}_{0, z}^{\lambda+1, \mu+1, \eta+1, p}(m) f(z)}, z \in \mathbb{U} .
$$

Differentiating logarithmically with respect to $z$ the above relation and using (5) we obtain

$$
\begin{gathered}
\frac{z p^{\prime}(z)}{p(z)}=(p-m-\mu) \frac{\mathrm{M}_{0, z}^{\lambda+1, \mu+1, \eta+1, p}(m) f(z)}{\mathrm{M}_{0, z}^{\lambda, \mu, \eta, p}(m) f(z)}- \\
-(p-m-\mu-1) \frac{\mathrm{M}_{0, z}^{\lambda+2, \mu+2, \eta+2, p}(m) f(z)}{\mathrm{M}_{0, z}^{\lambda+1, \mu+1, \eta+1, p}(m) f(z)}-1, z \in \mathbb{U} .
\end{gathered}
$$

Now we apply Lemma 1 with $\theta(w)=\beta w+\gamma w^{2}$ and $\varphi(w)=\frac{\zeta}{w}$. Clearly, $\theta$ is analytic in $\mathbb{C}$, $\varphi$ is analytic in $\mathbb{C}^{*}$ and $\varphi(w) \neq 0$ for $w \in \mathbb{C}^{*}$. Also, let $\psi(z)=z q^{\prime}(z) \varphi(q(z))=\zeta \frac{z q^{\prime}(z)}{q(z)}, z \in \mathbb{U}$, and $h(z)=\beta q(z)+\gamma q^{2}(z)+\zeta \frac{z q^{\prime}(z)}{q(z)}, z \in \mathbb{U}$. Since $\psi(0)=0, \psi^{\prime}(0) \neq 0$ and

$$
\operatorname{Re}\left(1+\frac{z q^{\prime \prime}(z)}{q^{\prime}(z)}-\frac{z q^{\prime}(z)}{q(z)}\right)>0, z \in \mathbb{U}
$$

so $\psi$ is starlike function in $\mathbb{U}$, and by (6)

$$
\operatorname{Re} \frac{z h^{\prime}(z)}{\psi(z)}=\operatorname{Re}\left[1+\frac{\beta}{\zeta} q(z)+\frac{2 \gamma}{\zeta} q^{2}(z)+\frac{z q^{\prime \prime}(z)}{q^{\prime}(z)}-\frac{z q^{\prime}(z)}{q(z)}\right]>0, z \in \mathbb{U} .
$$

Thus, according to the Lemma 1 , from (8) it follows that $p \prec q$ and $q$ is the best dominant, which completes our proof. 
Corollary 1. Assume that (6) holds with $q(z)=\frac{1+A z}{1+B z}, z \in \mathbb{U}$, with $-1 \leq B<A \leq 1$, and $f \in \mathcal{A}(p)$. If

$$
\Omega_{\lambda, \mu, \eta, p, m}(\beta, \gamma, \zeta) f(z) \prec \beta \frac{1+A z}{1+B z}+\gamma\left(\frac{1+A z}{1+B z}\right)^{2}+\zeta \frac{(A-B) z}{(1+A z)(1+B z)}, z \in \mathbb{U},
$$

then

$$
\frac{\mathrm{M}_{0, z}^{\lambda, \mu, \eta, p}(m) f(z)}{\mathrm{M}_{0, z}^{\lambda+1, \mu+1, \eta+1, p}(m) f(z)} \prec \frac{1+A z}{1+B z}, z \in \mathbb{U},
$$

and $q$ is the best dominant of (9).

Corollary 2. Assume that (6) holds with $q(z)=\left(\frac{1+z}{1-z}\right)^{\varepsilon}, z \in \mathbb{U}$, with $0<\varepsilon \leq 1$, and $f \in \mathcal{A}(p)$. If

$$
\Omega_{\lambda, \mu, \eta, p, m}(\beta, \gamma, \zeta) f(z) \prec \beta\left(\frac{1+z}{1-z}\right)^{\varepsilon}+\gamma\left(\frac{1+z}{1-z}\right)^{2 \varepsilon}+\zeta \frac{2 \varepsilon z}{\left(1-z^{2}\right)}, z \in \mathbb{U},
$$

then

$$
\frac{\mathrm{M}_{0, z}^{\lambda, \mu, \eta, p}(m) f(z)}{\mathrm{M}_{0, z}^{\lambda+1, \mu+1, \eta+1, p}(m) f(z)} \prec\left(\frac{1+z}{1-z}\right)^{\varepsilon}, z \in \mathbb{U}
$$

and $q$ is the best dominant of (10).

Corollary 3. Putting $\varepsilon=1$ in Corollary 2 we obtain the following special case:

Assume that (6) holds with $q(z)=\frac{1+z}{1-z}, z \in \mathbb{U}$, and $f \in \mathcal{A}(p)$. If

$$
\Omega_{\lambda, \mu, \eta, p, m}(\beta, \gamma, \zeta) f(z) \prec \beta \frac{1+z}{1-z}+\gamma\left(\frac{1+z}{1-z}\right)^{2}+\zeta \frac{2 z}{\left(1-z^{2}\right)}, z \in \mathbb{U},
$$

then

$$
\operatorname{Re} \frac{\mathrm{M}_{0, z}^{\lambda, \mu, \eta, p}(m) f(z)}{\mathrm{M}_{0, z}^{\lambda+1, \mu+1, \eta+1, p}(m) f(z)}>0, z \in \mathbb{U},
$$

and $q$ is the best dominant of (11).

Corollary 4. Assume that (6) holds with $q(z)=e^{A z}, z \in \mathbb{U}$, with $|A|<\pi$, and $f \in \mathcal{A}(p)$. If

$$
\Omega_{\lambda, \mu, \eta, p, m}(\beta, \gamma, \zeta) f(z) \prec \beta e^{A z}+\gamma e^{2 A z}+\zeta A z, z \in \mathbb{U},
$$

then

$$
\frac{\mathrm{M}_{0, z}^{\lambda, \mu, \eta, p}(m) f(z)}{\mathrm{M}_{0, z}^{\lambda+1, \mu+1, \eta+1, p}(m) f(z)} \prec e^{A z}, z \in \mathbb{U},
$$

and $q$ is the best dominant of (12).

Corollary 5. Let $a, b \in \mathbb{C}^{*}$ such that $|2 a b-1| \leq 1$ or $|2 a b+1| \leq 1$, let $f \in \mathcal{A}(p)$ and suppose that (6) holds for $q(z)=(1-z)^{-2 a b}, z \in \mathbb{U}$, with $a, b \in \mathbb{C}^{*}$. If

$$
\Omega_{\lambda, \mu, \eta, p, m}(\beta, \gamma, \zeta) f(z) \prec \frac{\beta}{(1-z)^{2 a b}}+\frac{\gamma}{(1-z)^{4 a b}}+\zeta \frac{2 a b z}{1-z}, z \in \mathbb{U},
$$

then

$$
\frac{\mathrm{M}_{0, z}^{\lambda, \mu, \eta, p}(m) f(z)}{\mathrm{M}_{0, z}^{\lambda+1, \mu+1, \eta+1, p}(m) f(z)} \prec(1-z)^{-2 a b}, z \in \mathbb{U},
$$

and $q$ is the best dominant of (13). 
Corollary 6. Suppose that (6) holds for $q(z)=(1+B z)^{\frac{\chi(A-B)}{B}}, z \in \mathbb{U}$, with $-1 \leq B<A \leq$ $1, B \neq 0, \chi \in \mathbb{C}^{*}$, such that $\left|\frac{\chi(A-B)}{B}-1\right| \leq 1$ or $\left|\frac{\chi(A-B)}{B}+1\right| \leq 1$. If $f \in \mathcal{A}(p)$ and

$$
\Omega_{\lambda, \mu, \eta, p, m}(\beta, \gamma, \zeta) f(z) \prec \beta(1+B z)^{\frac{\chi(A-B)}{B}}+\gamma(1+B z)^{\frac{2 \chi(A-B)}{B}}+\zeta \frac{(A-B) z}{1+B z}, z \in \mathbb{U},
$$

then

$$
\frac{\mathrm{M}_{0, z}^{\lambda, \mu, \eta, p}(m) f(z)}{\mathrm{M}_{0, z}^{\lambda+1, \mu+1, \eta+1, p}(m) f(z)} \prec(1+B z)^{\frac{\chi(A-B)}{B}}, z \in \mathbb{U},
$$

and $q$ is the best dominant of (14).

Using similar arguments to the proof of Theorem 1, we obtain the following theorem.

Theorem 2. Let $q$ be a convex function in $\mathbb{U}$ with $q(0)=1, q(z) \neq 0$ for all $z \in \mathbb{U}$ and $\operatorname{Re} \vartheta>0, \vartheta \in \mathbb{C}^{*}$. Let $f \in \mathcal{A}(p)$ and denote

$$
\begin{aligned}
& \Delta_{\lambda, \mu, \eta, p, m}(\vartheta) f(z):=\frac{\mathrm{M}_{0, z}^{\lambda, \mu, \eta, p}(m) f(z)}{\mathrm{M}_{0, z}^{\lambda+1, \mu+1, \eta+1, p}(m) f(z)}\left\{1+\vartheta(p-m-\mu) \frac{\mathrm{M}_{0, z}^{\lambda+1, \mu+1, \eta+1, p}(m) f(z)}{\mathrm{M}_{0, z}^{\lambda, \mu, \eta, p}(m) f(z)}-\right. \\
&\left.-\vartheta\left[(p-m-\mu-1) \frac{\mathrm{M}_{0, z}^{\lambda+2, \mu+2, \eta+2, p}(m) f(z)}{\mathrm{M}_{0, z}^{\lambda+1, \mu+1, \eta+1, p}(m) f(z)}+1\right]\right\}, z \in \mathbb{U} .
\end{aligned}
$$

If

$$
\Delta_{\lambda, \mu, \eta, p, m}(\vartheta) f(z) \prec q(z)+\vartheta z q^{\prime}(z), z \in \mathbb{U}
$$

then

$$
\frac{\mathrm{M}_{0, z}^{\lambda, \mu, \eta, p}(m) f(z)}{\mathrm{M}_{0, z}^{\lambda+1, \mu+1, \eta+1, p}(m) f(z)} \prec q(z), z \in \mathbb{U},
$$

and $q$ is the best dominant of (16).

Taking $m=0$ in Theorem 2 we obtain the following result due to Amsheri and Zharkova ([3, Theorem 2.1])

Corollary 7. Let $q$ be a convex function in $\mathbb{U}$ with $q(0)=1, q(z) \neq 0$ for all $z \in \mathbb{U}$ and $\operatorname{Re} \vartheta>0, \vartheta \in \mathbb{C}^{*}$, and denote

$$
\widetilde{\Delta}_{\lambda, \mu, \eta, p}(\vartheta):=\Delta_{\lambda, \mu, \eta, p, 0}(\vartheta)
$$

If $f \in \mathcal{A}(p)$ and

$$
\widetilde{\Delta}_{\lambda, \mu, \eta, p}(\vartheta) f(z) \prec q(z)+\vartheta z q^{\prime}(z), z \in \mathbb{U},
$$

then

$$
\frac{\mathcal{M}_{0, z}^{\lambda, \mu, \eta, p} f(z)}{\mathcal{M}_{0, z}^{\lambda+1, \mu+1, \eta+1, p} f(z)} \prec q(z), z \in \mathbb{U},
$$

and $q$ is the best dominant of (18). 
Theorem 3. Let $q$ be univalent in $\mathbb{U}$ with $q(0)=1, q(z) \neq 0$ for all $z \in \mathbb{U}, \frac{z q^{\prime}(z)}{q(z)}$ be starlike univalent in $\mathbb{U}$, and suppose that

$$
\operatorname{Re}\left[1+\frac{\sigma}{\xi} q(z)+\frac{2 \tau}{\xi} q^{2}(z)+\frac{z q^{\prime \prime}(z)}{q^{\prime}(z)}-\frac{z q^{\prime}(z)}{q(z)}\right]>0, z \in \mathbb{U},\left(\sigma, \tau \in \mathbb{C}, \xi \in \mathbb{C}^{*}\right) .
$$

Let $f \in \mathcal{A}(p)$ and denote

$$
\begin{gathered}
\Phi_{\lambda, \mu, \eta, p, m}(\sigma, \tau, \xi) f(z):=\frac{\sigma\left[\mathrm{M}_{0, z}^{\lambda, \mu, \eta, p}(m) f(z)\right]^{2}}{\delta(p, m) z^{p-m}\left[\mathrm{M}_{0, z}^{\lambda+1, \mu+1, \eta+1, p}(m) f(z)\right]}+ \\
+\frac{\tau\left[\mathrm{M}_{0, z}^{\lambda, \mu, \eta, p}(m) f(z)\right]^{4}}{\left[\delta(p, m) z^{p-m}\right]^{2}\left[\mathrm{M}_{0, z}^{\lambda+1, \mu+1, \eta+1, p}(m) f(z)\right]^{2}}+\xi\left[2(p-m-\mu) \frac{\mathrm{M}_{0, z}^{\lambda+1, \mu+1, \eta+1, p}(m) f(z)}{\mathrm{M}_{0, z}^{\lambda, \mu, \eta, p}(m) f(z)}-\right. \\
\left.-(p-m-\mu-1) \frac{\mathrm{M}_{0, z}^{\lambda+2, \mu+2, \eta+2, p}(m) f(z)}{\mathrm{M}_{0, z}^{\lambda+1, \mu+1, \eta+1, p}(m) f(z)}-(p-m-\mu+1)\right], z \in \mathbb{U} .
\end{gathered}
$$

If

$$
\Phi_{\lambda, \mu, \eta, p, m}(\sigma, \tau, \xi) f(z) \prec \sigma q(z)+\tau q^{2}(z)+\xi \frac{z q^{\prime}(z)}{q(z)}, z \in \mathbb{U}
$$

then

$$
\frac{\left[\mathrm{M}_{0, z}^{\lambda, \mu, \eta, p}(m) f(z)\right]^{2}}{\delta(p, m) z^{p-m}\left[\mathrm{M}_{0, z}^{\lambda+1, \mu+1, \eta+1, p}(m) f(z)\right]} \prec q(z), z \in \mathbb{U}
$$

and $q$ is the best dominant of (21).

Proof. Let

$$
p(z)=\frac{\left[\mathrm{M}_{0, z}^{\lambda, \mu, \eta, p}(m) f(z)\right]^{2}}{\delta(p, m) z^{p-m}\left[\mathrm{M}_{0, z}^{\lambda+1, \mu+1, \eta+1, p}(m) f(z)\right]}, z \in \mathbb{U} .
$$

We have

$$
\begin{gathered}
\frac{z p^{\prime}(z)}{p(z)}=2(p-m-\mu) \frac{\mathrm{M}_{0, z}^{\lambda+1, \mu+1, \eta+1, p}(m) f(z)}{\mathrm{M}_{0, z}^{\lambda, \mu, \eta, p}(m) f(z)}- \\
-(p-m-\mu-1) \frac{\mathrm{M}_{0, z}^{\lambda+2, \mu+2, \eta+2, p}(m) f(z)}{\mathrm{M}_{0, z}^{\lambda+1, \mu+1, \eta+1, p}(m) f(z)}-(p-m-\mu+1), z \in \mathbb{U} .
\end{gathered}
$$

Now we apply Lemma 1 with $\theta(w)=\sigma w+\tau w^{2}$ and $\varphi(w)=\frac{\xi}{w}$. Clearly, $\theta$ is analytic in $\mathbb{C}, \varphi$ is analytic in $\mathbb{C}^{*}$ and that $\varphi(w) \neq 0$ for $w \in \mathbb{C}^{*}$. Also, let $\psi(z)=z q^{\prime}(z) \varphi(q(z))=\xi \frac{z q^{\prime}(z)}{q(z)}, z \in \mathbb{U}$, and $h(z)=\sigma q(z)+\tau q^{2}(z)+\xi \frac{z q^{\prime}(z)}{q(z)}, z \in \mathbb{U}$. Since $\psi(0)=0, \psi^{\prime}(0) \neq 0$, and

$$
\operatorname{Re}\left(1+\frac{z q^{\prime \prime}(z)}{q^{\prime}(z)}-\frac{z q^{\prime}(z)}{q(z)}\right)>0, z \in \mathbb{U}
$$


implies that $\psi$ is starlike in $\mathbb{U}$ and by the assumption (19) we have

$$
\operatorname{Re} \frac{z h^{\prime}(z)}{\psi(z)}=\operatorname{Re}\left[1+\frac{\sigma}{\xi} q(z)+\frac{2 \tau}{\xi} q^{2}(z)+\frac{z q^{\prime \prime}(z)}{q^{\prime}(z)}-\frac{z q^{\prime}(z)}{q(z)}\right]>0, z \in \mathbb{U}
$$

Therefore, according to Lemma 1, the subordination (21) implies $p \prec q$ and $q$ is the best dominant.

Using similar arguments like in the proof of Theorem 3, we obtain the following theorem.

Theorem 4. Let $q$ be convex in $\mathbb{U}$ with $q(0)=1, q(z) \neq 0$ for all $z \in \mathbb{U}$ and $\operatorname{Re} \chi>0$, $\chi \in \mathbb{C}^{*}$. For $f \in \mathcal{A}(p)$ denote

$$
\begin{gathered}
\Psi_{\lambda, \mu, \eta, p, m}(\chi) f(z):=\frac{\left[\mathrm{M}_{0, z}^{\lambda, \mu, \eta, p}(m) f(z)\right]^{2}}{\delta(p, m) z^{p-m}\left[\mathrm{M}_{0, z}^{\lambda+1, \mu+1, \eta+1, p}(m) f(z)\right]} \times \\
\times\left[1+2 \chi(p-m-\mu) \frac{\mathrm{M}_{0, z}^{\lambda+1, \mu+1, \eta+1, p}(m) f(z)}{\mathrm{M}_{0, z}^{\lambda, \mu, \eta, p}(m) f(z)}-\right. \\
\left.-\chi(p-m-\mu-1) \frac{\mathrm{M}_{0, z}^{\lambda+2, \mu+2, \eta+2, p}(m) f(z)}{\mathrm{M}_{0, z}^{\lambda+1, \mu+1, \eta+1, p}(m) f(z)}-\chi(p-m-\mu+1)\right], z \in \mathbb{U} .
\end{gathered}
$$

If

$$
\Psi_{\lambda, \mu, \eta, p, m}(\chi) f(z) \prec q(z)+\chi z q^{\prime}(z), z \in \mathbb{U}
$$

then

$$
\frac{\left[\mathrm{M}_{0, z}^{\lambda, \mu, \eta, p}(m) f(z)\right]^{2}}{\delta(p, m) z^{p-m}\left[\mathrm{M}_{0, z}^{\lambda+1, \mu+1, \eta+1, p}(m) f(z)\right]} \prec q(z), z \in \mathbb{U},
$$

and $q$ is the best dominant of (23).

Taking $m=0$ in Theorem 4 we obtain the following result of Amsheri and Zharkova [3, Theorem 2.4]:

Corollary 8. Let $q$ be convex in $\mathbb{U}$ with $q(0)=1, q(z) \neq 0$ for all $z \in \mathbb{U}$ and $\operatorname{Re} \chi>0$, $\chi \in \mathbb{C}^{*}$, and denote

$$
\widetilde{\Psi}_{\lambda, \mu, \eta, p}(\chi):=\Psi_{\lambda, \mu, \eta, p, 0}(\chi) .
$$

If $f \in \mathcal{A}(p)$ and

$$
\widetilde{\Psi}_{\lambda, \mu, \eta, p}(\chi) f(z) \prec q(z)+\chi z q^{\prime}(z), z \in \mathbb{U},
$$

then

$$
\frac{\left[\mathcal{M}_{0, z}^{\lambda, \mu, \eta, p} f(z)\right]^{2}}{z^{p}\left[\mathcal{M}_{0, z}^{\lambda+1, \mu+1, \eta+1, p} f(z)\right]} \prec q(z), z \in \mathbb{U}
$$

and $q$ is the best dominant of (25). 


\section{Superordination results.}

Theorem 5. Let $q$ be analytic and univalent function in $\mathbb{U}$ with $q(0)=1, q(z) \neq 0$ for all $z \in \mathbb{U}, \frac{z q^{\prime}(z)}{q(z)}$ be starlike univalent in $\mathbb{U}$, and assume that

$$
\operatorname{Re}\left[\frac{\beta}{\zeta} q(z)+\frac{2 \gamma}{\zeta} q^{2}(z)\right]>0, z \in \mathbb{U},\left(\beta, \gamma \in \mathbb{C}, \zeta \in \mathbb{C}^{*}\right)
$$

For $f \in \mathcal{A}(p)$ suppose that

$$
0 \neq \frac{\mathrm{M}_{0, z}^{\lambda, \mu, \eta, p}(m) f(z)}{\mathrm{M}_{0, z}^{\lambda+1, \mu+1, \eta+1, p}(m) f(z)} \in \mathcal{H}[q(0), 1] \cap \mathcal{Q}
$$

and suppose that $\Omega_{\lambda, \mu, \eta, p, m}(\beta, \gamma, \xi) f$ defined by $(7)$ is univalent in $\mathbb{U}$. If $f$ satisfies the following superordination

$$
\beta q(z)+\gamma q^{2}(z)+\zeta \frac{z q^{\prime}(z)}{q(z)} \prec \Omega_{\lambda, \mu, \eta, p, m}(\beta, \gamma, \zeta) f(z), z \in \mathbb{U},
$$

then

$$
q(z) \prec \frac{\mathrm{M}_{0, z}^{\lambda, \mu, \eta, p}(m) f(z)}{\mathrm{M}_{0, z}^{\lambda+1, \mu+1, \eta+1, p}(m) f(z)}, z \in \mathbb{U},
$$

and $q$ is the best subordinant of (27).

Proof. Let

$$
p(z)=\frac{\mathrm{M}_{0, z}^{\lambda, \mu, \eta, p}(m) f(z)}{\mathrm{M}_{0, z}^{\lambda+1, \mu+1, \eta+1, p}(m) f(z)}, z \in \mathbb{U} .
$$

We apply Lemma 2 with $\theta(w)=\beta w+\gamma w^{2}$ and $\varphi(w)=\frac{\zeta}{w}$. Clearly, $\theta$ is analytic in $\mathbb{C}, \varphi$ is analytic in $\mathbb{C}^{*}$ and that $\varphi(w) \neq 0$ for $w \in \mathbb{C}^{*}$. Letting $\psi(z)=z q^{\prime}(z) \varphi(q(z))=\zeta \frac{z q^{\prime}(z)}{q(z)}, z \in \mathbb{U}$, the condition

$$
\operatorname{Re}\left(1+\frac{z q^{\prime \prime}(z)}{q^{\prime}(z)}-\frac{z q^{\prime}(z)}{q(z)}\right)>0, z \in \mathbb{U}
$$

implies that $\psi$ is a starlike function in $\mathbb{U}$ and by the assumption (26) we have

$$
\operatorname{Re} \frac{\theta^{\prime}(q(z))}{\varphi(q(z))}=\operatorname{Re}\left[\frac{\beta}{\zeta} q(z)+\frac{2 \gamma}{\zeta} q^{2}(z)\right]>0, z \in \mathbb{U} .
$$

Thus, from Lemma 2 the subordination (27) implies $q \prec p$ and $q$ best subordinant of (27).

Using similar arguments to the proof of Theorem 5, we obtain the following theorem:

Theorem 6. Let $q$ be an analytic and convex function in $\mathbb{U}$ with $q(0)=1, q(z) \neq 0$ for all $z \in \mathbb{U}$, and $\operatorname{Re} \vartheta>0, \vartheta \in \mathbb{C}^{*}$. For $f \in \mathcal{A}(p)$ suppose that

$$
0 \neq \frac{\mathrm{M}_{0, z}^{\lambda, \mu, \eta, p}(m) f(z)}{\mathrm{M}_{0, z}^{\lambda+1, \mu+1, \eta+1, p}(m) f(z)} \in \mathcal{H}[q(0), 1] \cap \mathcal{Q}
$$

and suppose that $\Delta_{\lambda, \mu, \eta, p, m}(\vartheta) f$ defined by (15) is univalent in $\mathbb{U}$. If

$$
q(z)+\vartheta z q^{\prime}(z) \prec \Delta_{\lambda, \mu, \eta, p, m}(\vartheta) f(z), z \in \mathbb{U},
$$


then

$$
q(z) \prec \frac{\mathrm{M}_{0, z}^{\lambda, \mu, \eta, p}(m) f(z)}{\mathrm{M}_{0, z}^{\lambda+1, \mu+1, \eta+1, p}(m) f(z)}, z \in \mathbb{U},
$$

and $q$ is the best subordinant of (28).

Taking $m=0$ in Theorem 5 we obtain the following result of Amsheri and Zharkova [3, Theorem 2.2].

Corollary 9. Let $q$ be an analytic and convex function in $\mathbb{U}$ with $q(0)=1, q(z) \neq 0$ for all $z \in \mathbb{U}$, and $\operatorname{Re} \vartheta>0, \vartheta \in \mathbb{C}^{*}$. For $f \in \mathcal{A}(p)$ suppose that

$$
0 \neq \frac{\mathcal{M}_{0, z}^{\lambda, \mu, \eta, p} f(z)}{\mathcal{M}_{0, z}^{\lambda+1, \mu+1, \eta+1, p} f(z)} \in \mathcal{H}[q(0), 1] \cap \mathcal{Q}
$$

and suppose that $\widetilde{\Delta}_{\lambda, \mu, \eta, p}(\vartheta) f$ defined by $(17)$ is univalent in $\mathbb{U}$. If

$$
q(z)+\vartheta z q^{\prime}(z) \prec \widetilde{\Delta}_{\lambda, \mu, \eta, p}(\vartheta) f(z), z \in \mathbb{U},
$$

then

$$
q(z) \prec \frac{\mathcal{M}_{0, z}^{\lambda, \mu, \eta, p} f(z)}{\mathcal{M}_{0, z}^{\lambda+1, \mu+1, \eta+1, p} f(z)}, z \in \mathbb{U},
$$

and $q$ is the best subordinant of (29).

Using arguments similar to that in the proof of Theorem 5 we obtain the following two theorems:

Theorem 7. Let $q$ be analytic and univalent function in $\mathbb{U}$ with $q(0)=1, q(z) \neq 0$ for all $z \in \mathbb{U}$ and $\frac{z q^{\prime}(z)}{q(z)}$ be starlike univalent in $\mathbb{U}$, such that

$$
\operatorname{Re}\left[\frac{\sigma}{\xi} q(z)+\frac{2 \tau}{\xi} q^{2}(z)\right]>0, z \in \mathbb{U}
$$

For $f \in \mathcal{A}(p)$ suppose that

$$
0 \neq \frac{\left[\mathrm{M}_{0, z}^{\lambda, \mu, \eta, p}(m) f(z)\right]^{2}}{\delta(p, m) z^{p-m}\left[\mathrm{M}_{0, z}^{\lambda+1, \mu+1, \eta+1, p}(m) f(z)\right]} \in \mathcal{H}[q(0), 1] \cap \mathcal{Q} .
$$

and suppose that $\Phi_{\lambda, \mu, \eta, p, m}(\sigma, \tau, \xi) f$ defined by $(20)$ is univalent in $\mathbb{U}$. If

$$
\sigma q(z)+\tau q^{2}(z)+\xi \frac{z q^{\prime}(z)}{q(z)} \prec \Phi_{\lambda, \mu, \eta, p, m}(\sigma, \tau, \xi) f(z), z \in \mathbb{U}, \quad\left(\sigma, \tau \in \mathbb{C}, \xi \in \mathbb{C}^{*}\right)
$$

then

$$
q(z) \prec \frac{\left[\mathrm{M}_{0, z}^{\lambda, \mu, \eta, p}(m) f(z)\right]^{2}}{\delta(p, m) z^{p-m}\left[\mathrm{M}_{0, z}^{\lambda+1, \mu+1, \eta+1, p}(m) f(z)\right]}, z \in \mathbb{U},
$$

and $q$ is the best subordinant of (31). 
Theorem 8. Let $q$ be an analytic and convex function in $\mathbb{U}$ with $q(0)=1, q(z) \neq 0$ for all $z \in \mathbb{U}$, and $\operatorname{Re} \chi>0, \chi \in \mathbb{C}^{*}$. For $f \in \mathcal{A}(p)$ suppose that

$$
0 \neq \frac{\left[\mathrm{M}_{0, z}^{\lambda, \mu, \eta, p}(m) f(z)\right]^{2}}{\delta(p, m) z^{p-m}\left[\mathrm{M}_{0, z}^{\lambda+1, \mu+1, \eta+1, p}(m) f(z)\right]} \in \mathcal{H}[q(0), 1] \cap \mathcal{Q}
$$

and suppose $\Psi_{\lambda, \mu, \eta, p, m}(\chi) f$ defined by $(22)$ is univalent in $\mathbb{U}$. If

$$
q(z)+\chi z q^{\prime}(z) \prec \Psi_{\lambda, \mu, \eta, p, m}(\chi) f(z), z \in \mathbb{U}
$$

then

$$
q(z) \prec \frac{\left[\mathrm{M}_{0, z}^{\lambda, \mu, \eta, p}(m) f(z)\right]^{2}}{\delta(p, m) z^{p-m}\left[\mathrm{M}_{0, z}^{\lambda+1, \mu+1, \eta+1, p}(m) f(z)\right]}, z \in \mathbb{U},
$$

and $q$ is the best subordinant of (32).

Taking $m=0$ in Theorem 7 we obtain the following result due to Amsheri and Zharkova [3, Theorem 2.5]:

Corollary 10. Let $q$ be analytic and convex function in $\mathbb{U}$ with $q(0)=1, q(z) \neq 0$ for all $z \in \mathbb{U}$, and $\operatorname{Re} \chi>0, \chi \in \mathbb{C}^{*}$. For $f \in \mathcal{A}(p)$ suppose that

$$
0 \neq \frac{\left[\mathcal{M}_{0, z}^{\lambda, \mu, \eta, p} f(z)\right]^{2}}{z^{p}\left[\mathcal{M}_{0, z}^{\lambda+1, \mu+1, \eta+1, p} f(z)\right]} \in \mathcal{H}[q(0), 1] \cap \mathcal{Q}
$$

and suppose that $\widetilde{\Psi}_{\lambda, \mu, \eta, p}(\chi) f$ defined by $(24)$ is univalent in $\mathbb{U}$. If

$$
q(z)+\chi z q^{\prime}(z) \prec \widetilde{\Psi}_{\lambda, \mu, \eta, p}(\chi) f(z), z \in \mathbb{U},
$$

then

$$
q(z) \prec \frac{\left[\mathcal{M}_{0, z}^{\lambda, \mu, \eta, p} f(z)\right]^{2}}{z^{p}\left[\mathcal{M}_{0, z}^{\lambda+1, \mu+1, \eta+1, p} f(z)\right]}, z \in \mathbb{U},
$$

and $q$ is the best subordinant of (33).

4. Sandwich results. Combining Theorem 1 and Theorem 5 we obtain the following sandwich-type theorem.

Theorem 9. Let $q_{1}$ and $q_{2}$ be univalent functions in $\mathbb{U}$ with $q_{1}(0)=q_{2}(0)=1$, such that $q_{1}(z) \neq 0, q_{2}(z) \neq 0$ for all $z \in \mathbb{U}$. Assume that $\frac{z q_{1}^{\prime}(z)}{q_{1}(z)}$ and $\frac{z q_{2}^{\prime}(z)}{q_{2}(z)}$ are starlike univalent in $\mathbb{U}$, and (6) and (26) hold for $q_{2}$ and $q_{1}$, respectively. For $f \in \mathcal{A}(p)$ suppose that

$$
0 \neq \frac{\mathrm{M}_{0, z}^{\lambda, \mu, \eta, p}(m) f(z)}{\mathrm{M}_{0, z}^{\lambda+1, \mu+1, \eta+1, p}(m) f(z)} \in \mathcal{H}[1,1] \cap \mathcal{Q}
$$


and suppose that $\Omega_{\lambda, \mu, \eta, p, m}(\beta, \gamma, \zeta) f$ defined by $(7)$ is univalent in $\mathbb{U}$. If

$$
\begin{gathered}
\beta q_{1}(z)+\gamma q_{1}^{2}(z)+\xi \frac{z q_{1}^{\prime}(z)}{q_{1}(z)} \prec \Omega_{\lambda, \mu, \eta, p, m}(\beta, \gamma, \zeta) f(z) \prec \\
\prec \beta q_{2}(z)+\gamma q_{2}^{2}(z)+\xi \frac{z q_{2}^{\prime}(z)}{q_{2}(z)}, z \in \mathbb{U}, \quad\left(\beta, \gamma \in \mathbb{C}, \zeta \in \mathbb{C}^{*}\right)
\end{gathered}
$$

then

$$
q_{1}(z) \prec \frac{\mathrm{M}_{0, z}^{\lambda, \mu, \eta, p}(m) f(z)}{\mathrm{M}_{0, z}^{\lambda+1, \mu+1, \eta+1, p}(m) f(z)} \prec q_{2}(z), z \in \mathbb{U},
$$

and $q_{1}$ and $q_{2}$ are respectively the best subordinant and best dominant of (34).

Combining Theorem 2 and Theorem 6 we obtain the following sandwich-type result:

Theorem 10. Let $q_{1}$ and $q_{2}$ be convex functions in $\mathbb{U}$ with $q_{1}(0)=q_{2}(0)=1$ such that $q_{1}(z) \neq 0, q_{2}(z) \neq 0$ for all $z \in \mathbb{U}$, and assume that $\operatorname{Re} \vartheta>0 \vartheta \in \mathbb{C}^{*}$. For $f \in \mathcal{A}(p)$ suppose that

$$
0 \neq \frac{\mathrm{M}_{0, z}^{\lambda, \mu, \eta, p}(m) f(z)}{\mathrm{M}_{0, z}^{\lambda+1, \mu+1, \eta+1, p}(m) f(z)} \in \mathcal{H}[1,1] \cap \mathcal{Q}
$$

and suppose that $\Delta_{\lambda, \mu, \eta, p, m}(\vartheta) f$ defined by (15) is univalent in $\mathbb{U}$. If

$$
q_{1}(z)+\vartheta z q_{1}^{\prime}(z) \prec \Delta_{\lambda, \mu, \eta, p, m}(\vartheta) f(z) \prec q_{2}(z)+\vartheta z q_{2}^{\prime}(z), z \in \mathbb{U},
$$

then

$$
q_{1}(z) \prec \frac{\mathrm{M}_{0, z}^{\lambda, \mu, \eta, p}(m) f(z)}{\mathrm{M}_{0, z}^{\lambda+1, \mu+1, \eta+1, p}(m) f(z)} \prec q_{2}(z), z \in \mathbb{U},
$$

and $q_{1}$ and $q_{2}$ are respectively the best subordinant and best dominant of (35).

Remark 3. Taking $m=0$ in Theorem 10 we obtain the result of Amsheri and Zharkova [3, Theorem 2.3].

Combining Theorem 3 and Theorem 7 we obtain the following sandwich-type result:

Theorem 11. Let $q_{1}$ and $q_{2}$ be univalent functions in $\mathbb{U}$ with $q_{1}(0)=q_{2}(0)=1$ such that $q_{1}(z) \neq 0, q_{2}(z) \neq 0$ for all $z \in \mathbb{U}$. Assume that $\frac{z q_{1}^{\prime}(z)}{q_{1}(z)}$ and $\frac{z q_{2}^{\prime}(z)}{q_{2}(z)}$ are starlike univalent in $\mathbb{U}$, and (19) and (30) hold for $q_{2}$ and $q_{1}$, respectively. For $f \in \mathcal{A}(p)$ suppose that

$$
0 \neq \frac{\left[\mathrm{M}_{0, z}^{\lambda, \mu, \eta, p}(m) f(z)\right]^{2}}{\delta(p, m) z^{p-m}\left[\mathrm{M}_{0, z}^{\lambda+1, \mu+1, \eta+1, p}(m) f(z)\right]} \in \mathcal{H}[1,1] \cap \mathcal{Q}
$$

and suppose that $\Phi_{\lambda, \mu, \eta, p, m}(\sigma, \tau, \xi) f$ defined by (20) is univalent in $\mathbb{U}$. If

$$
\begin{gathered}
\sigma q_{1}(z)+\tau q_{1}^{2}(z)+\xi \frac{z q_{1}^{\prime}(z)}{q_{1}(z)} \prec \Phi_{\lambda, \mu, \eta, p, m}(\sigma, \tau, \xi) f(z) \prec \\
\prec \sigma q_{2}(z)+\tau q_{2}^{2}(z)+\xi \frac{z q_{2}^{\prime}(z)}{q_{2}(z)}, z \in \mathbb{U}, \quad\left(\sigma, \tau \in \mathbb{C}, \xi \in \mathbb{C}^{*}\right)
\end{gathered}
$$


then

$$
q_{1}(z) \prec \frac{\left[\mathrm{M}_{0, z}^{\lambda, \mu, \eta, p}(m) f(z)\right]^{2}}{\delta(p, m) z^{p-m}\left[\mathrm{M}_{0, z}^{\lambda+1, \mu+1, \eta+1, p}(m) f(z)\right]} \prec q_{2}(z), z \in \mathbb{U},
$$

and $q_{1}$ and $q_{2}$ are respectively the best subordinant and best dominant of (36).

Combining Theorem 4 and Theorem 8 we obtain the following sandwich-type theorem:

Theorem 12. Let $q_{1}$ and $q_{2}$ be convex functions in $\mathbb{U}$ with $q_{1}(0)=q_{2}(0)=1$ such that $q_{1}(z) \neq 0, q_{2}(z) \neq 0$ for all $z \in \mathbb{U}$, and assume that $\operatorname{Re} \chi>0, \vartheta \in \mathbb{C}^{*}$. For $f \in \mathcal{A}(p)$ suppose that

$$
0 \neq \frac{\left[\mathrm{M}_{0, z}^{\lambda, \mu, \eta, p}(m) f(z)\right]^{2}}{\delta(p, m) z^{p-m}\left[\mathrm{M}_{0, z}^{\lambda+1, \mu+1, \eta+1, p}(m) f(z)\right]} \in \mathcal{H}[1,1] \cap \mathcal{Q}
$$

and suppose that $\Psi_{\lambda, \mu, \eta, p, m}(\chi) f$ defined by $(22)$ is univalent in $\mathbb{U}$. If

$$
q_{1}(z)+\vartheta z q_{1}^{\prime}(z) \prec \Psi_{\lambda, \mu, \eta, p, m}(\chi) f(z) \prec q_{2}(z)+\vartheta z q_{2}^{\prime}(z), z \in \mathbb{U},
$$

then

$$
q_{1}(z) \prec \frac{\left[\mathrm{M}_{0, z}^{\lambda, \mu, \eta, p}(m) f(z)\right]^{2}}{\delta(p, m) z^{p-m}\left[\mathrm{M}_{0, z}^{\lambda+1, \mu+1, \eta+1, p}(m) f(z)\right]} \prec q_{2}(z), z \in \mathbb{U},
$$

and $q_{1}$ and $q_{2}$ are respectively the best subordinant and best dominant of (37).

Remark 4. (i) Taking $m=0$ in Theorem 12 we obtain the result due to Amsheri and Zharkova;

(ii) Putting $\lambda=\mu$ in the above theorems we obtain new sandwich results for the operator $D_{z}^{\lambda}(m)$ defined by $(4)$.

\section{REFERENCES}

1. R.M. Ali, V. Ravichandran, K.M. Hussain, K.G. Subramanian, Differential sandwich theorems for certain analytic functions, Far East J. Math. Sci., 15 (2004), №1, 87-94.

2. S.M. Amsheri, V. Zharkova, Differential subordinations and superordinations for p-valent functions defined by fractional derivative operator, Demonstr. Math., 46 (2013), №3, 505-515.

3. S.M. Amsheri, V. Zharkova, Differential sandwich theorems of p-valent functions associated with a certain fractional derivative operator, Kragujevac J. Math., 35 (2011), №3, 387-398 .

4. M.K. Aouf, A.O. Mostafa, H.M. Zayed, Some characterizations of integral operators associated with certain classes of p-valent functions defined by the Srivastava-Saigo-Owa fractional differintegral operator, Complex Anal. Oper. Theory, 10 (2016), №6, 1267-1275.

5. M.K. Aouf, A.O. Mostafa, H.M. Zayed, Subordination and superordination properties of p-valent functions defined by a generalized fractional differintegral operator, Quaest. Math., 39 (2016) №4, 545-560.

6. M.K. Aouf, A.O. Mostafa, H.M. Zayed, On certain subclasses of multivalent functions defined by a generalized fractional differintegral operator, Afr. Mat., 28 (2017), №1-2, 99-107.

7. T. Bulboacă, Classes of first-order differential superordinations, Demonstr. Math., 35 (2002), №2, 287292. 
8. T. Bulboacă, A class of superordination-preserving integral operators, Indag. Math. (N.S.), 13 (2002), №3, 301-311.

9. T. Bulboacă, Differential subordinations and superordinations, new results, House of Scientific Boook Publ., Cluj-Napoca, 2005.

10. G.P. Goyal, J.K. Prajapat, A new class of analytic p-valent functions with negative coefficients and fractional calculus operators, Tamsui Oxf. J. Inf. Math. Sci., 20 (2004), №2, 175-186.

11. S.S. Miller, P.T. Mocanu, Differential subordinations: theory and applications, Series on Monographs and Textbooks in Pure and Appl. Math., V.255, Marcel Dekker, Inc., New York, 2000.

12. S.S. Miller, P.T. Mocanu, Subordinations of differential superordinations, Complex Var., 48 (2003) №10, $815-826$.

13. A.O. Mostafa, M.K. Aouf, H.M. Zayed, T. Bulboacă, Multivalent functions associated with SrivastavaSaigo-Owa fractional differintegral operator. Rev. R. Acad. Cienc. Exactas Fís. Nat. Ser. A Math. RACSAM, https://doi.org/10.1007/s13398-017-0436-1, /to appear/

14. S. Owa, On the distortion theorems I. Kyungpook Math. J., 18 (1978), 53-59.

15. W.C. Royster, On the univalence of a certain integral, Michigan Math. J., 12 (1965), №4, 385-387.

16. T.N. Shanmugam, V. Ravichandran, S. Sivasubramanian, Differential sandwich theorems for some subclasses of analytic functions, Aust. J. Math. Anal. Appl., 3 (2006), №1, 1-11.

Department of Mathematics, Faculty of Science Menofia University, Shebin Elkom, Egypt

hanaa_zayed42@yahoo.com

Faculty of Mathematics and Computer Science

Babeş-Bolyai University, Cluj-Napoca, Romania

bulboaca@math.ubbcluj.ro 